\title{
Ethical dilemmas in surgery: some philosophical reflections
}

\author{
Michael Lockwood University College, Oxford
}

\section{Editor's note}

In this paper Michael Lockwood makes some philosophical comments on the various ethical dilemmas of surgery detailed in the foregoing papers from the Surgeons' Conference. He highlights clinical trials, surgery for the aged and organs for transplants, all of which subjects are most topical and not only in medical circles. Dr Lockwood also touches upon the taboos of tribal culture in the Third World as well as use of paramedical staff in areas where fully trained personnel are at a premium. In the face of increasing public concern, he is encouraged to find a growing sensitivity amongst surgeons to the ethical implications of their work.

The distinguished surgeons represented in this volume have evidently thought deeply about the ethical problems arising within their profession. The issues they discuss are many and varied: we have heard about the ethics of clinical trials, informed consent (especially within tribal cultures or amongst those who, for one reason or another, are incompetent to make their own rational assessment), delegation of responsibility, the ethics of maintaining body function in brain-dead patients so as to provide organs for transplant, and nontreatment of the terminally ill, under conditions where little value would seem to attach to a surgically won increment in lifespan. These are topics which present both practical and philosophical challenges.

\section{Clinical trials}

Giertz's and Rudowski's timely discussions of clinical trials raise a host of problems. Two, at least, may bear restatement. First, the results of such trials are, inevitably, at their most convincing when the trials themselves are blind or double blind. This, by definition, requires to a certain degree, keeping the patient in the dark as to the exact nature of his treatment. To what extent, then, can this be consistent with the principle of obtaining the patient's informed consent: a requirement which would seem all the more pressing in regard to treatment which is largely untried? Secondly, is there not a constant danger, in clinical trials, of the doctor finding himself giving or withholding an certain form of treatment, according as the patient is assigned to the test or control group, when there is at least some evidence that the patient's own $\vec{\omega}$ interests are not best served thereby ? Giertz himself says that such trials are only ethically accept-⿳亠乛了 able 'where it is not possible from already available facts to decide which treatment is preferable'; butio if 'decide', here, is taken to cover presumptive evidence, I suspect that this would rule out ao substantial proportion of actual such trials. RudowskL emphasises 'the need to accumulate a largec number of patients', 'sufficient numbers of cases? [being] needed for statistical significance'. But is it compatible with a physician's contractual re sponsibilities to his patient deliberately to withhold from him - by way of making him a control - treat-응 ment that has very nearly proved its efficacy to thes desired level of statistical significance, especiallyo if the patient's prognosis is otherwise terminal : More obviously, is it compatible with a doctor' $\mathbb{D}$ responsibilities to replace a method of treatment of proven efficacy with a possibly hazardous substitute even if medical knowledge is extended thereby ? Notoriously, there are pressures so to do. Lord Smith has stressed the need for doctors to maintaing autonomy of judgment in the face of the collectivis demands of the state; by the same token, doctor must not be placed in a position where their duties? to their patients in the here and now becomes subordinate to the hypothetical benefits that mighe accrue from adding a decimal digit to the signifio cance level of the results of some medical experi- $\rightarrow$ ment. From a purely practical standpoint, the nees. for randomised trials of the ethically more questionable variety might be much diminished if doctors simply doing the best for their patients in the lighis of the information available to them - were tow supply detailed records which could then be colo lated and subjected to statistical analysis, possiblyo on an international scale.

\section{Surgery for the aged?}

I admired Reiss's balanced and humane discussion of the pros and cons of prolonging the lives of the aged by surgical intervention. I agree with him that the surgeon has no right, much less a duty, tion administer life prolonging treatment to the lucieo and competent' patient who simply does not wan 
it. The difficulties, as he himself points out, arise where, for one reason or another, it is impossible in any meaningful fashion - to consult the patient himself. Though considerations as to the quality of life must, as Reiss says, be used with extreme caution, it is difficult to see what other guide-line one can employ in such cases. It has been suggested, with some plausibility, that the right question to ask is whether the patient, were he sufficiently lucid to be consulted, would wish for such treatment. But in the case of the severely retarded or senile, it is often doubtful whether this question has any clear sense, much less how, if it does, the doctor is supposed to divine the answer. It should perhaps be stressed that the aged are often able to face the inevitability of death with considerably more equanimity than the surgeon, at the height of his physical and mental powers, can readily appreciate. There comes a point, certainly, at which massive technological intervention simply becomes an assault against the right to an easy and dignified death: a sentiment beautifully expressed by Lord Smith's quotation from King Lear. I quarrel only with Reiss's curious refusal to describe the wilful failure to administer potentially life prolonging surgery as 'passive euthanasia'. Surely it is precisely that. Or is the issue somehow so sensitive that we need a euphemism for a euphemism?

\section{The 'barefoot' doctor}

It is impossible not to sympathise with Cook in his desire to extend the availability of surgical procedures to poorer countries, without thereby degrading the profession. And he is obviously under no illusions as to the difficulty of reconciling these aims. Nevertheless, I suspect that sheer economic necessity will result in many poorer countries evolving medical services in which the 'barefoot doctor' or the 'paramedic' comes to perform many roles which, in the West (Nilsson's Sweden, for example), would be the exclusive province of the fully trained surgeon. Already, India had deployed large numbers of paramedics with considerable skill in removing cataracts, but little else in the way of medical expertise - and with tremendous success. Is the profession of surgery thereby degraded ? Possibly. But in the end, such procedures will be approved and adopted to the extent that people are helped by them. The 'harsh reality' of which Cook speaks is that, in a country with only, say, one general practitioner for every 20,000 people, and few facilities for training more, it is either the paramedic or nothing, the barefoot doctor or the witch doctor. The most we can reasonably hope, perhaps, is that the standards of surgery maintained in the West continue to serve as a guiding, though, for the poorest countries, probably unattainable, ideal.

\section{Social or religious taboos and 'consent'}

Ajayi has provided us with a vivid picture of the difficulties that arise in attempting to apply, to tribal cultures where ignorance, superstition and illiteracy are the norm, ethical standards tailored to the needs of Western societies. To the extent that obedience to the tribal chief or village headman is the major factor in determining preparedness to accept treatment, it seems eminently sensible to involve these local leaders, from the start, in the decision process. Where petty despotism cannot, without seriously undermining the social structure, be removed or bypassed, education can perhaps render it more benign. But although these cultures may present the medical practitioner with special problems, the same principle of informed consent surely must apply. To be sure, it may be difficult or impossible to get the prospective patient properly to understand what is at issue; he may, it is true, simply refer the decision to someone he perceives as in a position of authority; and doubtless he will be influenced by taboos and so forth. But then the third world hardly has a monopoly on ignorance: how capable are many patients in the West of making a well informed decision on what may, after all, be a highly technical matter ? Then again, how different, in principle is the patient in Western Europe or North America who refuses, on religious grounds, a certain form of treatment, from the African with his taboos ? And as for deferring to authority, is it not the case that a high proportion of patients, in the West, do just that in respect of the doctor or surgeon himself ? Reiss remarked, en passant, that it was only very rarely that his patients failed to take his advice. 'Informed' consent can only ever mean consent, by the patient, while possessed of as much relevant information as it is possible effectively to communicate to him, subject to the limitations of time, education and intelligence. And if the patient ends up refusing treatment because someone whose authority he respects tells him to, or because it offends against his religion, that may be regrettable. But I am sure Ajayi would agree that it is, after all, his right so to do.

\section{Organs for transplant}

I turn, finally, to Lord Smith's remarks concerning the maintenance of body function, in the now or soon to be brain dead, solely for the purpose of providing a supply of transplantable organs. There is, without doubt, something ghoulish in this conception; and it is this feeling, perhaps, that prompts Lord Smith to think that there is an ethical problem here. But provided it is a question of the prolongation merely of body function, and not, say, suffering, can there, on reflection, be any rational objection to this practice? Any lingering sense that there is can only, I should have thought, be evidence of doctors (and the public at large) having failed to 
accept with their hearts what they have already accepted with their heads: namely that a brain dead patient is, after all, dead. The heart may be beating, and the lungs respiring, but the body is nevertheless a corpse, not a living human being. In the end, the surgeon who has lost a patient might even find solace in the thought that his efforts have not been entirely in vain - if the preservation of body function, by his skill, can serve to enhance or even save the lives of others.
There is much that is thought provoking in foregoing essays, much that I have not even touched on in these brief remarks. Suffice it to say that i very reassuring for the layman to find, amongst surgeons, such sensitivity to the ethical implications of their work. With the advance in surgical scienfe, one finds that what once were questions largely for philosophical debate, turn with bewilder rapidity into matters of widespread public concern. 